PROCEEDINGS OF THE

AMERICAN MATHEMATICAL SOCIETY

Volume 128, Number 3, Pages 647-655

S 0002-9939(99)05418-0

Article electronically published on July 27, 1999

\title{
THE GLOBALLY IRREDUCIBLE REPRESENTATIONS OF SYMMETRIC GROUPS
}

\author{
ALEXANDER KLESHCHEV AND ALEXANDER PREMET
}

(Communicated by Ronald M. Solomon)

\begin{abstract}
Let $K$ be an algebraic number field and $\mathcal{O}$ be the ring of integers of $K$. Let $G$ be a finite group and $M$ be a finitely generated torsion free $\mathcal{O} G$-module. We say that $M$ is a globally irreducible $\mathcal{O} G$-module if, for every maximal ideal $\mathfrak{p}$ of $\mathcal{O}$, the $k_{\mathfrak{p}} G$-module $M \otimes \mathcal{O} k_{\mathfrak{p}}$ is irreducible, where $k_{\mathfrak{p}}$ stands for the residue field $\mathcal{O} / \mathfrak{p}$.

Answering a question of Pham Huu Tiep, we prove that the symmetric group $\Sigma_{n}$ does not have non-trivial globally irreducible modules. More precisely we establish that if $M$ is a globally irreducible $\mathcal{O} \Sigma_{n}$-module, then $M$ is an $\mathcal{O}$-module of rank 1 with the trivial or sign action of $\Sigma_{n}$.
\end{abstract}

\section{InTRODUCTION AND GENERALITIES}

The notion of global irreducibility was first introduced by J. G. Thompson in the course of constructing the sporadic group $F_{3}([9,10])$. He observed that there exists an even unimodular $\mathbb{Z}$-lattice $\Lambda$ of $\operatorname{rank} 248$ with Aut $(\Lambda) \cong \mathbb{Z}_{2} \times F_{3}$ such that the $\mathbb{F}_{p} F_{3}$-module $\Lambda / p \Lambda$ is irreducible for every prime $p$. Other interesting examples of the same nature were discovered later, and different (closely related) notions of global irreducibility were introduced. A good survey on the subject is [11].

Let $K$ be an algebraic number field and $\mathcal{O}$ be the ring of integers of $K$. Let $G$ be a finite group and $M$ be a finitely generated torsion free $\mathcal{O} G$-module. We say that $M$ is globally irreducible if, for every maximal ideal $\mathfrak{p}$ of $\mathcal{O}$, the $k_{\mathfrak{p}} G$-module $M \otimes_{\mathcal{O}} k_{\mathfrak{p}}$ is irreducible, where $k_{\mathfrak{p}}$ denotes the residue field $\mathcal{O} / \mathfrak{p}$. This definition seems to "include" all versions of global irreducibility introduced before.

Let $\Sigma_{n}$ denote the symmetric group on $n$ letters. The main result of this paper is the following theorem which answers a question posed by Pham Huu Tiep.

Theorem A. Let $M$ be a globally irreducible $\mathcal{O} \Sigma_{n}$-module. Then $M$ is an $\mathcal{O}$ module of rank 1 with the trivial or sign action of $\Sigma_{n}$.

Throughout the paper:

$\mathbb{N}=\{1,2, \ldots\}$ is the set of all natural numbers,

$F$ is an arbitrary field of characteristic $p>0$ (unless otherwise stated).

Received by the editors December 10, 1997 and, in revised form, April 15, 1998.

1991 Mathematics Subject Classification. Primary 20C30, 20C10.

Key words and phrases. Symmetric group, Specht module.

The authors thank G. Michler and A. Zalesskii who organized a conference on representations of finite groups in Bad-Honnef where this collaboration began, and the Volkswagen foundation for financial support. The first author was also supported by the NSF.

(C)1999 American Mathematical Society 
For $r, s \in \mathbb{N}$ we denote:

$$
\begin{aligned}
& {[r, s]=\{t \in \mathbb{N} \mid r \leq t \leq s\} ; \quad(r, s]=\{t \in \mathbb{N} \mid r<t \leq s\}} \\
& {[r, s)=\{t \in \mathbb{N} \mid r \leq t<s\} ; \quad(r, s)=\{t \in \mathbb{N} \mid r<t<s\} .}
\end{aligned}
$$

Given a partition $\lambda$ of $n$, we often gather together equal parts of $\lambda$ and write

$$
\lambda=\left(\lambda_{1}^{a_{1}}, \lambda_{2}^{a_{2}}, \ldots, \lambda_{k}^{a_{k}}\right),
$$

where $\lambda_{1}>\lambda_{2}>\cdots>\lambda_{k}>0, a_{i}>0$, and $a_{1} \lambda_{1}+a_{2} \lambda_{2}+\cdots+a_{k} \lambda_{k}=n$. A partition $\lambda$ is called p-regular if $a_{i}<p$ for all $i$. We denote by $\lambda^{\prime}$ the partition conjugate to $\lambda$ (the Young diagram of $\lambda^{\prime}$ is the transpose of the Young diagram of $\lambda)$. The symbol $(i, j)$ denotes the node in the $i$ th row and the $j$ th column of the Young diagram of $\lambda$, and $h(i, j)=h_{\lambda}(i, j)$ stands for the length of the hook of $\lambda$ corresponding to the node $(i, j)$. Note that the notation for the node $(i, j)$ should not be confused with the one for the interval $(i, j)$ as defined above! In what follows we do not distinguish between partitions and their Young diagrams.

We introduce some notions from the representation theory of symmetric groups. Details can be found in [6]. Let $S_{\mathbb{Z}}^{\lambda}$ denote the Specht module over $\mathbb{Z}$ corresponding to $\lambda$ (it carries a canonical $\mathbb{Z} \Sigma_{n}$-module structure). Given a field $F$ set $S_{F}^{\lambda}=$ $S_{\mathbb{Z}}^{\lambda} \otimes_{\mathbb{Z}} F$. It is well-known that if char $F=0$, then $S_{F}^{\lambda}$ is an irreducible $F \Sigma_{n}$-module. In general, the irreducible $F \Sigma_{n}$-module corresponding to a $p$-regular partition $\lambda$ is denoted by $D_{F}^{\lambda}$. Note that $D_{F}^{\lambda}$ is always a quotient of $S_{F}^{\lambda}$. Another fact which is going to be used without comments is that $\mathbb{F}_{p}$ is the splitting field for the group $\Sigma_{n}$. So the question of the irreducibility of $S_{F}^{\lambda}$ depends on the characteristic of $F$ only, not on $F$ itself.

We shall deduce Theorem A from the following

Theorem B. The Specht module $S_{\mathbb{Z}}^{\lambda}$ is globally irreducible if and only if $\lambda=(n)$ or $\left(1^{n}\right)$.

We now explain why Theorem B implies Theorem A.

Let $M$ be a globally irreducible $\mathcal{O} \Sigma_{n}$-module, and $M_{K}=M \otimes \mathcal{O} K$. Then $M_{K}$ is a direct sum of Specht modules over $K$ (see [6, Theorem 4.12]). It follows that there exists a $\mathbb{Z} \Sigma_{n}$-module $M_{\mathbb{Z}}$ such that $M_{K} \cong M_{\mathbb{Z}} \otimes_{\mathbb{Z}} K$ as $K \Sigma_{n}$-modules. Moreover, $M_{\mathbb{Z}}$ can be chosen to be a direct sum of Specht modules over $\mathbb{Z}$. By a theorem of Brauer-Nesbitt, the $k_{\mathfrak{p}} \Sigma_{n}$-modules $M \otimes_{\mathcal{O}} k_{\mathfrak{p}}$ and $M_{\mathbb{Z}} \otimes_{\mathbb{Z}} k_{\mathfrak{p}}$ have the same composition factors (see, e.g., [2, Theorem 82.1]). We claim that $M_{\mathbb{Z}}$ is a globally irreducible $\mathbb{Z} \Sigma_{n}$-module isomorphic to a Specht module $S_{\mathbb{Z}}^{\lambda}$ for some partition $\lambda$ of $n$. Indeed, if $\bar{M}=M_{\mathbb{Z}} \otimes_{\mathbb{Z}} \mathbb{F}_{p}$ is reducible for some prime $p$, then, for each maximal ideal $\mathfrak{q}$ of $\mathcal{O}$ such that $\mathfrak{q} \cap \mathbb{Z}=p \mathbb{Z}$, the $k_{\mathfrak{q}} \Sigma_{n}$-module $M_{\mathbb{Z}} \otimes_{\mathbb{Z}} k_{\mathfrak{q}} \cong \bar{M} \otimes_{\mathbb{F}_{p}} k_{\mathfrak{q}}$ is reducible. Also, if $M_{\mathbb{Z}}$ is a direct sum of more than one Specht module over $\mathbb{Z}$, then, for each maximal ideal $\mathfrak{p}$ of $\mathcal{O}$, the $k_{\mathfrak{p}} \Sigma_{n}$-module $M_{\mathbb{Z}} \otimes_{\mathbb{Z}} k_{\mathfrak{p}}$ is reducible again. This establishes the claim in view of the global irreducibility of $M$ and the result of Brauer-Nesbitt mentioned above.

Now suppose we have proved Theorem B. Then the $\mathbb{Z}$-rank of $M_{\mathbb{Z}}$ is 1 . So $\operatorname{dim} M_{K}=1$, i.e. $M$ is an $\mathcal{O}$-module of rank 1 . Hence the subgroup $A_{n}=\Sigma_{n}^{\prime}$ acts trivially on $M$, and Theorem A follows. 


\section{Some REPRESENTATION THEORY AND SOME NUMBER THEORY}

The following result known as Carter's conjecture was proved by James and Murphy $[5,7]$. Recall that the $p$-exponent of an integer $n$ is the largest power of the prime $p$ dividing $n$.

Proposition 2.1. Let $\lambda$ be a p-regular partition of $n$. Then $S_{F}^{\lambda}$ is irreducible if and only if the p-exponents of the hook lengths of the Young diagram of $\lambda$ are constant along the columns.

Our first lemma is a straightforward consequence of Proposition 2.1.

Lemma 2.2. Assume that both $\lambda$ and $\lambda^{\prime}$ are $p$-regular partitions of $n$. If there is a hook in the Young diagram of $\lambda$ whose length is divisible by $p$, then $S_{F}^{\lambda}$ is reducible.

Proof. Suppose that $S_{F}^{\lambda}$ is irreducible and some $h_{\lambda}(i, j)$ is divisible by $p$. In view of Proposition 2.1 all hook lengths in the $j$ th column of the Young diagram of $\lambda$ should be divisible by $p$. But this is impossible for the bottom hook in the $j$ th column, since $\lambda^{\prime}$ is $p$-regular.

From now on we fix an arbitrary partition $\lambda$ of $n$. Let

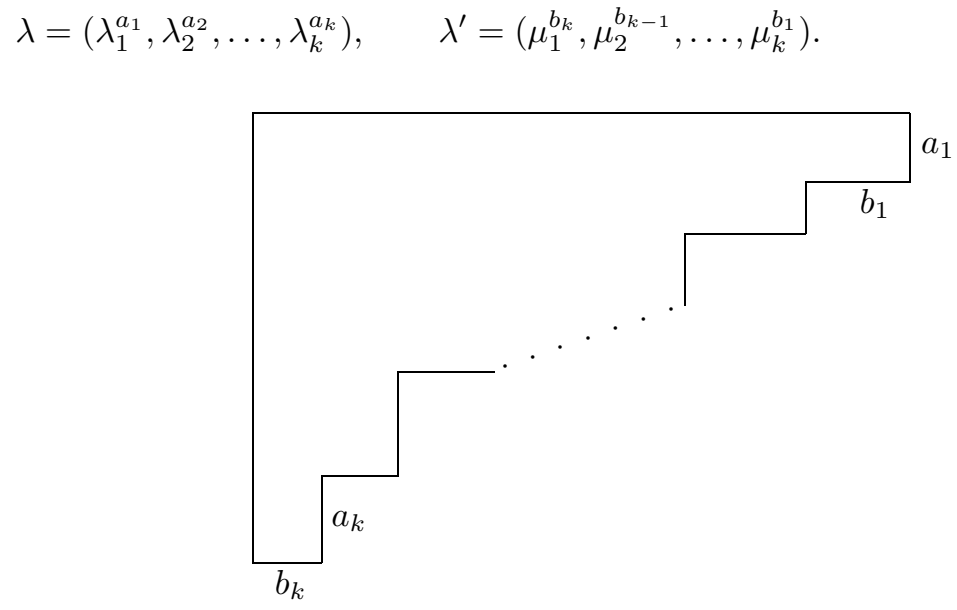

Put

$$
\begin{aligned}
A_{s} & =\left(\sum_{i=1}^{s} a_{i}, \lambda_{s}\right), s=1,2, \ldots, k \\
B_{r} & =\left(\sum_{i=1}^{r-1} a_{i}+1, \lambda_{r}+1\right), r=1,2, \ldots, k+1
\end{aligned}
$$

$\left(\lambda_{k+1}\right.$ is interpreted as 0$)$. Then $A_{1}, \ldots, A_{k}$ and $B_{1}, \ldots, B_{k+1}$ are all the removable and the addable nodes for $\lambda$, respectively. We denote by $\lambda(r, s)$ the partition of $n$ whose Young diagram is obtained from that of $\lambda$ by removing $A_{s}$ and adding $B_{r}$. This partition is not well defined exactly in the following cases:

(a) $r=s, a_{r}=1$;

(b) $r=s+1, b_{s}=1$. 
So when we write $\lambda(r, s)$ we assume that neither (a) nor (b) occurs. Clearly, $\lambda(r, s) \triangleright$ $\lambda$ if $r \leq s$ and $\lambda(r, s) \triangleleft \lambda$ if $r>s$.

We make use of the so-called p-ladders introduced by James in [4]. Those are, roughly speaking, straight lines with slope $(p-1)$. More precisely, the $u$-th $p$-ladder is defined as the following set of nodes:

$$
L_{u}:=\{(i, j) \mid i, j \in \mathbb{N}, i+(p-1)(j-1)=u\} .
$$

If we slide the nodes of $\lambda$ along the $p$-ladders as far up as they can go, then we obtain a new partition $\lambda^{R}$, called the p-regularization of $\lambda$. It is proved in [4] that $\lambda^{R}$ is well defined and that $\lambda$ is $p$-regular if and only if $\lambda=\lambda^{R}$.

Proposition $2.3([4])$. $D_{F}^{\lambda^{R}}$ occurs in $S_{F}^{\lambda}$ with multiplicity 1 , and if $D_{F}^{\mu}$ is a composition factor of $S_{F}^{\lambda}$, then $\mu \unrhd \lambda^{R}$.

Lemma 2.4. Suppose that $\lambda$ and $\lambda(r, s)$ are p-regular. Let $A_{s}$ belong to the $v$-th $p$-ladder, and $B_{r}$ belong to the $u$-th $p$-ladder. Then $\lambda(r, s) \triangleright \lambda$ if and only if $u>v$.

Proof. It was mentioned above that $\lambda(r, s) \triangleright \lambda$ if and only if $r \leq s$. By definition,

$$
v=\sum_{i=1}^{s} a_{i}+(p-1)\left(\lambda_{s}-1\right) \text { and } u=\sum_{i=1}^{r-1} a_{i}+1+(p-1) \lambda_{r} .
$$

Now $u>v$ is equivalent to

$$
\sum_{i=1}^{s} a_{i}-\sum_{i=1}^{r-1} a_{i}<(p-1)\left(\lambda_{r}-\lambda_{s}\right)+p .
$$

Suppose $r>s$ and $u>v$. Then

$$
\sum_{i=s+1}^{r-1} a_{i}>(p-1)\left(\lambda_{s}-\lambda_{r}\right)-p .
$$

Note that $(p-1)\left(\lambda_{s}-\lambda_{r}\right) \geq(p-1)(r-s)$, and

$$
\sum_{i=s+1}^{r-1} a_{i} \leq(r-1-s)(p-1),
$$

since $\lambda$ is $p$-regular. Therefore (2) holds if and only if $a_{i}=p-1$ for $s+1 \leq i \leq r-1$ and $\lambda_{s}-\lambda_{r}=r-s$. If $r=s+1$, this gives $b_{s}=\lambda_{s}-\lambda_{s+1}=1$, so $\lambda(r, s)$ is not defined. If $r>s+1$, we get $a_{r-1}=p-1$, and $b_{r-1}=1$. Thus $\lambda(r, s)$ is not $p$-regular, giving a contradiction. Hence $r>s$ implies $u \leq v$.

Let $r \leq s$. Then

$$
\sum_{i=r}^{s} a_{i} \leq(s-r+1)(p-1)<(s-r)(p-1)+p \leq\left(\lambda_{r}-\lambda_{s}\right)(p-1)+p,
$$

which in combination with (1) forces $u>v$. Thus we have proved that $r>s$ implies $u \leq v$ and $r \leq s$ implies $u>v$, as desired.

Lemma 2.5. Suppose $A_{s}$ belongs to the $v$-th p-ladder and $B_{r}$ belongs to the $u$-th p-ladder.

(i) If $u=v$, then $\lambda(r, s)^{R}=\lambda^{R}$.

(ii) If $u \neq v$, then $\lambda(r, s)^{R}$ is obtained from $\lambda^{R}$ by moving the bottom removable node of $\lambda^{R}$ on the $p$-ladder $L_{v}$ to the top addable node for $\lambda^{R}$ on the $p$-ladder $L_{u}$ (and such nodes exist). 
Proof. This follows from the definition of $p$-regularization.

Denote

$$
A_{r, s}=\sum_{i=r}^{s} a_{i}, \quad B_{r, s}=\sum_{j=r}^{s} b_{j} .
$$

(The sums are interpreted as 0 if $r>s$.)

Lemma 2.6. Let $r \leq s$. Then $\lambda(r, s)^{R} \triangleright \lambda^{R}$ if and only if $A_{r, s}<p+(p-1) B_{r, s-1}$.

Proof. Let $A_{s}$ belong to the $v$-th $p$-ladder and $B_{r}$ belong to the $u$-th $p$-ladder. By Lemma 2.5, $\lambda(r, s)^{R}$ is obtained from $\lambda^{R}$ by moving a removable node on the $v$ th $p$-ladder to an addable node on the $u$-th $p$-ladder. Since both $\lambda^{R}$ and $\lambda(r, s)^{R}$ are $p$-regular, Lemma 2.4 implies that $\lambda(r, s)^{R} \triangleright \lambda^{R}$ if and only if $u>v$, which is equivalent to

$$
A_{1, s}+(p-1)\left(\lambda_{s}-1\right)<A_{1, r-1}+1+(p-1) \lambda_{r} .
$$

Now the result follows from the equality $\lambda_{r}-\lambda_{s}=\sum_{j=r}^{s-1} b_{j}$. [1].

The following proposition is a special case of the main result of Carter-Payne

Proposition 2.7 ([1]). (i) Let $1 \leq r \leq s \leq k$. If $p$ divides $A_{r, s}+B_{r, s-1}$, then $\operatorname{Hom}_{F \Sigma_{n}}\left(S_{F}^{\lambda(r, s)}, S_{F}^{\lambda}\right) \neq 0$.

(ii) Assume that $k \geq 2, b_{2} \geq 2$, and $p$ is odd. Let

$$
\begin{array}{r}
\mu=\left(\lambda_{1}+2, \lambda_{1}^{a_{1}-1}, \lambda_{2}^{a_{2}-1}, \lambda_{2}-2, \lambda_{3}^{a_{3}}, \ldots, \lambda_{k}^{a_{k}}\right) . \\
\text { If } p \text { divides } a_{1}+a_{2}+b_{1}+1 \text {, then } \operatorname{Hom}_{F \Sigma_{n}}\left(S_{F}^{\mu}, S_{F}^{\lambda}\right) \neq 0 .
\end{array}
$$

Our further arguments rely heavily on the following consequence of Proposition 2.7.

Lemma 2.8. Let $\lambda$ be a partition of $n$.

(i) Suppose there exist $r, s \in \mathbb{N}$ with $1 \leq r<s \leq k$ such that $p$ divides $A_{r, s}+$ $B_{r, s-1}$ and $A_{r, s}<(p-1) B_{r, s-1}+p$. Then $S_{F}^{\lambda}$ is reducible.

(ii) Suppose that $k \geq 2, b_{2} \geq 2, a_{1}+a_{2}<(p-1)\left(b_{1}+2\right)+1$, and $p$ is odd. If $p$ divides $a_{1}+a_{2}+b_{1}+1$, then $S_{F}^{\lambda}$ is reducible.

Proof. (i) By Proposition 2.7(i),

$$
\operatorname{Hom}_{F \Sigma_{n}}\left(S_{F}^{\lambda(r, s)}, S_{F}^{\lambda}\right) \neq 0 .
$$

By Lemma 2.6, our assumption guarantees that $\lambda(r, s)^{R} \triangleright \lambda^{R}$. If $S_{F}^{\lambda}$ is irreducible, then it is isomorphic to $D_{F}^{\lambda^{R}}$ (see Proposition 2.3). On the other hand, by the same proposition, each composition factor $D_{F}^{\mu}$ of $S_{F}^{\lambda(r, s)}$ satisfies $\mu \unrhd \lambda(r, s)^{R} \triangleright \lambda^{R}$; hence the Hom-space above should be zero. This contradiction proves (i).

For (ii), let $\mu$ be as in Proposition 2.7(ii). Then the Young diagram of $\mu$ can be obtained from that of $\lambda$ by moving two nodes from the $\left(a_{1}+a_{2}\right)$ th row of the Young diagram of $\lambda$ to the first row. Observe that $\mu$ is well defined as $b_{2} \geq 2$. By Proposition 2.7(ii),

$$
\operatorname{Hom}_{F \Sigma_{n}}\left(S_{F}^{\mu}, S_{F}^{\lambda}\right) \neq 0 .
$$

Let $C=\left(a_{1}+a_{2}, \lambda_{2}\right), D=\left(a_{1}+a_{2}, \lambda_{2}-1\right), X=\left(1, \lambda_{1}+1\right)$, and $Y=\left(1, \lambda_{1}+2\right)$. The Young diagram of $\mu$ can be obtained from that of $\lambda$ in two steps. First we 
remove $C$ and add $X$ to get a partition $\nu$. Then we remove $D$ from the Young diagram of $\nu$ and add $Y$. By our assumption,

$$
a_{1}+a_{2}+b_{1}+1<p\left(b_{1}+1\right)+p .
$$

As $p$ divides $a_{1}+a_{2}+b_{1}+1$, we must have

$$
a_{1}+a_{2}+b_{1}+1 \leq p\left(b_{1}+1\right)
$$

whence $a_{1}+a_{2}<(p-1) b_{1}+p$. So $\nu^{R} \triangleright \lambda^{R}$ by Lemma 2.6 (one should keep in mind that $\nu=\lambda(1,2)$ ). Applying Lemma 2.6 for the second time we obtain $\mu^{R} \triangleright \nu^{R} \triangleright \lambda^{R}$. Now we finish the proof as in part (i).

The next three lemmas are versions (and generalizations) of the well-known Chebyshev's results on prime numbers.

Lemma 2.9. Let $n \in \mathbb{N}$. Then the interval $(n, 2 n+1]$ contains at least two primes.

Proof. It is known [3, p. 21] that, for $n \geq 21$, the number of primes on the interval $(n, 2 n+1]$ exceeds $3 n /(5 \ln n)$. Clearly, $3 n /(5 \ln n)$ is greater than 2 if $n \geq 21$. Now check the result for $n=1,2, \ldots, 20$.

Lemma 2.10. Let $n$ be an integer greater than 1 . Then $(n, 2 n)$ contains a prime.

Proof. This is the famous "Bertrand's postulate" proved by Chebyshev (see, e.g., [3, p. 21]).

Lemma 2.11. Let $n \in \mathbb{N}$. Then $\left(n, \frac{3}{2} n+2\right]$ contains a prime.

Proof. For $n \geq 25$, the interval $\left(n, \frac{6}{5} n\right)$ contains a prime (see [8, p. 192]). Now check the result for $n=1,2, \ldots, 24$.

Remark. It is easy to see that Lemma 2.9 also follows from the fact that the interval $\left(n, \frac{6}{5} n\right)$ contains a prime $([8$, p. 192]).

\section{Proof of Theorem B}

Given a partition $\lambda$ of $n$ set

$$
A=\max _{1 \leq i \leq k}\left\{a_{i}\right\}, \quad B=\max _{1 \leq j \leq k}\left\{b_{j}\right\}
$$

(the parameters $a_{i}, b_{j}$, and $k$ are defined in Section 2). We wish to prove that $S_{\mathbb{Z}}^{\lambda}$ is globally irreducible if and only if $\lambda \in\left\{(n),\left(1^{n}\right)\right\}$. It is well-known that $\left(S_{F}^{\lambda}\right)^{*} \cong\left(S_{F}^{\lambda^{\prime}}\right) \otimes$ sgn. Therefore, $S_{\mathbb{Z}}^{\lambda}$ is globally irreducible if and only if $S_{\mathbb{Z}}^{\lambda^{\prime}}$ is. So no generality is lost by assuming that $B \geq A$. Let $f \in\{1,2, \ldots, k\}$ be such that $b_{f}=B$.

Lemma 3.1. Suppose that $S_{\mathbb{Z}}^{\lambda}$ is globally irreducible, and $B \geq A$. Then any integer in the interval $\left[1, A_{1, f}+B_{1, f}-1\right]$ is a hook length of $\lambda$.

Proof. We prove by the downward induction on $d=f, f-1, \ldots, 1$ that any integer in the interval $\left[1, A_{d, f}+B_{d, f}-1\right]$ is a hook length of $\lambda$.

If $d=f$, we have $A_{d, f}+B_{d, f}-1=a_{f}+b_{f}-1$. In this case, it suffices to note that $h\left(A_{1, f}, B_{f, k}-v\right)=v+1, v=0,1, \ldots, b_{f}-1$, and $h\left(A_{1, f}-w, B_{f+1, k}+1\right)=b_{f}+w$, $w=0,1, \ldots, a_{f}-1$.

Now suppose that $1 \leq d<f$. By the inductive hypothesis, the hook lengths of $\lambda$ cover the interval $\left[1, A_{d+1, f}+B_{d+1, f}-1\right]$. Note that

$$
h\left(A_{1, d}, B_{f, k}-v\right)=A_{d+1, f}+B_{d, f-1}+1+v, \quad v=0,1, \ldots, b_{f}-1,
$$


and

$$
h\left(A_{1, d}-w, B_{f+1, k}+1\right)=A_{d+1, f}+B_{d, f}+w, \quad w=0,1, \ldots, a_{d}-1 .
$$

Thus each number in the union

$$
\left[1, A_{d+1, f}+B_{d+1, f}-1\right] \cup\left[A_{d+1, f}+B_{d, f-1}+1, A_{d, f}+B_{d, f}-1\right]
$$

is a hook length of $\lambda$. If $A_{d+1, f}+B_{d+1, f}>A_{d+1, f}+B_{d, f-1}$, the union (3) equals the interval [1, $\left.A_{d, f}+B_{d, f}-1\right]$, as desired. Otherwise we have

$$
A_{d+1, f}+B_{d+1, f} \leq A_{d+1, f}+B_{d, f-1}
$$

which is equivalent to $b_{f} \leq b_{d}$. Since $b_{f}=B$, we conclude that $b_{d}=b_{f}=B$ yielding

$$
A_{d+1, f}+B_{d+1, f}=A_{d+1, f}+B_{d, f-1}=: x
$$

that is, the union (3) equals $\left[1, A_{d, f}+B_{d, f}-1\right] \backslash\{x\}$. Note that

$$
A_{d, f}+B_{d, f} \geq a_{d}+a_{f}+b_{d}+b_{f} \geq 2+2 B \text {. }
$$

Hence $\left[1, A_{d, f}+B_{d, f}-1\right]$ contains $(B, 2 B+1]$. By Lemma 2.9 , there is a prime $p>B$ which is a hook length of $\lambda$. Now Lemma 2.2 implies that $S_{\mathbb{Z}}^{\lambda}$ is not globally irreducible. This accomplishes the induction step.

Lemma 3.2. Suppose $S_{\mathbb{Z}}^{\lambda}$ is globally irreducible. Then either $n=1$ or $B \geq 2$.

Proof. Suppose $B=1$ and $n \geq 2$. Then $\lambda$ is the "staircase partition", and $k \geq 2$. Hence 3 is a hook length for $\lambda$. Now, by Lemma 2.2, $S_{F}^{\lambda}$ is reducible in characteristic 3.

In view of Corollary 3.2 we may (and will) assume that our globally irreducible Specht module $S_{\mathbb{Z}}^{\lambda}$ satisfies the condition $B \geq \max \{2, A\}$. Let $q$ denote the maximal prime in the interval $[2, B]$.

Lemma 3.3. Assume that $S_{\mathbb{Z}}^{\lambda}$ is globally irreducible, and $B \geq \max \{2, A\}$. If $f=k$, then $\lambda=(n)$.

Proof. By Lemma 3.1, the hook lengths of $\lambda$ cover the interval [1, $\left.A_{1, k}+B_{1, k}-1\right]$. We claim that $a_{i}<q$ for all $i \leq k$. Indeed, suppose $a_{i} \geq q$ for some $i$. Then

$$
A_{1, k}+B_{1, k}-1 \geq b_{k}+a_{i}-1 \geq 2 q-1 \text {. }
$$

So the hook lengths of $\lambda$ cover $(q, 2 q)$. By Lemma 2.10, there is a prime $p>q$ such that $p$ is a hook length of $\lambda$. Now Lemma 2.2 leads us to a contradiction (it applies as $p>B$ ). Thus $a_{i}<q$ for all $i$, that is, $\lambda$ is $q$-regular. But then $\lambda$ should satisfy Carter's condition (see Proposition 2.1).

Observe that

$$
h\left(A_{1, k}, b_{k}-(q-1)\right)=q .
$$

Therefore, $a_{k}=1$ since otherwise

$$
h\left(A_{1, k}-1, b_{k}-(q-1)\right)=q+1
$$

and $\lambda$ does not satisfy Carter's condition. If $k=1$, this implies that $\lambda=(n)$. So suppose $k>1$. Then

$$
h\left(A_{1, k}-1, b_{k}-(q-1)\right)=q+1+b_{k-1} .
$$

Now Carter's condition forces $b_{k-1} \equiv-1(\bmod q)$. In particular, $b_{k-1} \geq q-1$. This gives $A_{1, k}+B_{1, k}-1 \geq 2+b_{k}+b_{k-1}-1 \geq 2 q$, so that $\left[1, A_{1, k}+B_{1, k}-1\right] \supseteq(q, 2 q)$. Again Lemmas 2.10 and 2.2 lead us to a contradiction completing the proof. 
Now we are in a position to finish the proof of Theorem B.

Theorem 3.4. Suppose $S_{\mathbb{Z}}^{\lambda}$ is globally irreducible and $B \geq \max \{2, A\}$. Then $\lambda=$ $(n)$.

Proof. By Lemma 3.3, we may assume that $f<k$. Applying Lemma 2.8(i) with $r=f, s=f+1$ shows that

$$
a_{f}+a_{f+1} \geq(p-1) b_{f}+p
$$

for any prime $p$ dividing $a_{f}+a_{f+1}+b_{f}$.

If an odd prime divides $a_{f}+a_{f+1}+b_{f}$, then (4) implies that $a_{f}+a_{f+1} \geq 2 b_{f}+3$. In this case, either $a_{f}>b_{f}$ or $a_{f+1}>b_{f}$ contrary to the choice of $b_{f}$. Thus we must have

$$
a_{f}+a_{f+1}+b_{f}=2^{m} .
$$

Combining this with (4) we get

$$
a_{f}+a_{f+1} \geq b_{f}+2 .
$$

Assume $f>1$. Applying Lemma 2.8(i) with $r=f-1, s=f+1$ gives that

$$
a_{f-1}+a_{f}+a_{f+1} \geq(p-1)\left(b_{f-1}+b_{f}\right)+p
$$

for any prime $p$ dividing $a_{f-1}+a_{f}+a_{f+1}+b_{f}+b_{f-1}$. If an odd prime divides this number, then (7) yields

$$
a_{f-1}+a_{f}+a_{f+1} \geq 2 b_{f-1}+2 b_{f}+3 .
$$

By Lemma 3.1, the hook lengths of $\lambda$ cover $\left[1, a_{f}+a_{f-1}+b_{f}+b_{f-1}-1\right]$. If

$$
a_{f}+a_{f-1}+b_{f}+b_{f-1}-1 \geq 2 b_{f}-1 \text {, }
$$

then $\left[1, a_{f}+a_{f-1}+b_{f}+b_{f-1}-1\right] \supseteq(q, 2 q)$. So again Lemma 2.10 ensures that there exists a prime $p_{1}>q$ which is a hook length of $\lambda$, and again Lemma 2.2 shows that this contradicts the global irreducibility of $S_{\mathbb{Z}}^{\lambda}$. Thus

$$
a_{f}+a_{f-1}+b_{f}+b_{f-1}-1<2 b_{f}-1
$$

which is equivalent to

$$
2 b_{f}-b_{f-1}>a_{f}+a_{f-1}+b_{f} .
$$

However, this violates (8) as $b_{f} \geq a_{f+1}$. So we conclude that

$$
a_{f-1}+a_{f}+a_{f+1}+b_{f}+b_{f-1}=2^{M}
$$

for some $M>m$. From (5) and (9) we have

$$
a_{f-1}+b_{f-1}=2^{M}-2^{m}=2^{m}\left(2^{M-m}-1\right) \geq 2^{m} .
$$

Therefore, either $a_{f-1} \geq 2^{m-1}$ or $b_{f-1} \geq 2^{m-1}$. Now (6) says that

$$
b_{f}<\frac{1}{2}\left(a_{f}+a_{f+1}+b_{f}\right)=2^{m-1} .
$$

So $b_{f}<a_{f-1}$ or $b_{f}<b_{f-1}$ contrary to the choice of $f$.

Thus we may assume that $f=1$. If $b_{2} \geq 2$, then applying Lemma 2.8(ii) gives that there exists an odd prime $p$ for which

$$
a_{1}+a_{2} \geq(p-1)\left(b_{1}+2\right)+1
$$

(indeed, $a_{1}+a_{2}+b_{1}+1$ is odd by (5)). As $p \geq 3$, (10) implies that $a_{1}+a_{2} \geq 2 b_{1}+5$, whence either $a_{1}>b_{1}$ or $a_{2}>b_{1}$. Since this is impossible, we must have $b_{2}=1$. 
Applying Lemma 2.8(i) to $\lambda^{\prime}$ instead of $\lambda$ we obtain that

$$
1+b_{1} \geq(p-1) a_{2}+p
$$

for any prime $p$ dividing $a_{2}+b_{1}+1$. If there is an odd prime with this property, then $1+b_{1} \geq 2 a_{2}+3$. Combining this with (6) (which is $a_{1}+a_{2} \geq b_{1}+2$ as $f=1$ ) we get $a_{1} \geq a_{2}+4$. Therefore, $a_{1}+a_{1}-4 \geq a_{1}+a_{2} \geq b_{1}+2$ yielding

$$
a_{1} \geq \frac{b_{1}}{2}+3 \text {. }
$$

So $\left[1, a_{1}+b_{1}-1\right] \supset\left(b_{1}, \frac{3}{2} b_{1}+2\right]$. Due to Lemmas 2.11 and 3.1 one of the hooks of the Young diagram of $\lambda$ has length equal to a prime greater than $q$. By Lemma 2.2, this is impossible. Thus $a_{2}+b_{1}+1=2^{s}$. In view of (5) we also have $a_{1}+a_{2}+b_{1}=2^{m}$. So

$$
a_{1}-1=2^{s}\left(2^{m-s}-1\right),
$$

and $m \geq s$. If $m>s$, we get $b_{1}<2^{s} \leq a_{1}-1$ which is false as $1=f$. If $m=s$, we conclude that $a_{1}=1$. But then $a_{1}+a_{2} \geq b_{1}+2$ forces $a_{2}>b_{1}$ leading us to a contradiction with the choice of $b_{f}$. This contradiction proves the theorem.

\section{REFERENCES}

[1] R. W. Carter and M. T. J. Payne, On homomorphisms between Weyl modules and Specht modules, Math. Proc. Camb. Phil. Soc. 87 (1980), 419-425. MR 81h:20048

[2] C. W. Curtis and I. Reiner, Representation theory of finite groups and associative algebras, John Wiley and Sons (Interscience), New York, 1962. MR 26:2519

[3] W. and F. Ellison, Prime numbers, John Wiley and Sons, Hermann, 1985. MR 87a:11082

[4] G. D. James, On the decomposition matrices of the symmetric groups, II, J. Algebra 43 (1976), 45-54. MR 55:3057b

[5] G. D. James, On a conjecture of Carter concerning irreducible Specht modules. Math. Proc. Camb. Phil. Soc. 83 (1978), 11-17. MR 57:3234

[6] G. D. James, The representation theory of the symmetric groups, Springer Lecture Notes 682, Berlin, Heidelberg, New York, 1978. MR 80g:20019

[7] G. D. James and G. E. Murphy, The determinant of the Gram matrix for a Specht module. J. Algebra 59 (1979), 222-235. MR 82j:20025

[8] P. Ribbenboim, The book of prime number records, Springer-Verlag, New York, Berlin, Heidelberg, 1988.

[9] J. G. Thompson, Finite groups and even lattices. J. Algebra 38 (1976), 523-524. MR 53:3108

[10] J. G. Thompson, A simple subgroup of $E_{8}(3)$. In: N. Iwahori (ed.), Finite groups symposium, Japan Soc. for promotion of Science, 1976, pp. 113-116.

[11] Pham Huu Tiep, Globally irreducible representations of finite groups and integral lattices, Geometriae Dedicata 64 (1997), 85-123. MR 98e:20011

Department of Mathematics, University of Oregon, Eugene, Oregon 97403

E-mail address: klesh@math.uoregon.edu

Department of Mathematics, University of Manchester, Oxford Road, Manchester, M13 9PL, United Kingdom

E-mail address: sashap@ma.man.ac.uk 